\title{
SOME NONEXISTENCE THEOREMS FOR INITIAL-BOUNDARY VALUE PROBLEMS WITH NONLINEAR BOUNDARY CONSTRAINTS ${ }^{1}$
}

\author{
H. A. LEVINE AND L. E. PAYNE
}

ABSTRACT. The present work extends previous results of the authors [4] on nonexistence of solutions of classes of second order equations subject to certain nonlinear boundary constraints and appropriate hypotheses on the data. Similar results are derived here for more general classes of higher order equations.

1. Introduction. In an earlier paper [4] the authors studied certain classes of nonlinear initial-boundary value problems for the heat and wave equations. In this investigation special types of nonlinear boundary constraints were imposed. It was shown that for these problems no solution with initial data in a specific class can exist for all time. In the present paper we derive similar results for more general classes of higher order equations.

Let $M$ denote a formally selfadjoint differential operator of order $2 m$ defined on a bounded region $D$ of $R^{n}$. The coefficients of the operator are assumed to possess derivatives of the order indicated in the formal expression for the operator. We could (see [1], [4]) reduce the differentiability requirements and work with weak solutions, but since the arguments are formally the same regardless of whether we consider weak or strong solutions, we shall limit our discussion here to classical solutions.

For elements $u$ and $v$ in the domain of the operator $M$ we may write the following expression:

$$
\int_{D}[v M u-u M v] d x=\sum_{j=0}^{m-1} \oint_{\partial D}\left\{N_{j}(v) Q_{j}(u)-N_{j}(u) Q_{j}(v)\right\} d s
$$

where $N_{j}$ and $Q_{j}$ are linear differential operators on the boundary; for defi-

Received by the editors September 26, 1973.

AMS (MOS) subject classifications (1970). Primary 35B05, 35B40, 35F 30, $35 \mathrm{~K} 60,35 \mathrm{~L} 99$.

Key words and phrases. Nonexistence theorems, initial-boundary value problems, nonlinear boundary constraints, concavity methods, differential inequalities, finite escape time.

1 This research was supported in part by NSF Grant GP-33031X1. 
niteness we assume that $N_{j}$ is a boundary operator containing derivatives up to order $j$ while $Q_{j}$ is of order $2 m-1-j$. The forms of $N_{j}$ and $Q_{j}$ are not uniquely determined by (1.1). For example, in the the ory of elastic plates where $M=\Delta^{2}$ (the biharmonic operator), the boundary operators may depend on a parameter usually referred to as Poisson's ratio. The symbol $\partial D$ denotes the boundary of $D$.

We assume further that the differential operators $M, N$, and $Q$ are such that the quantity $A(v, u)$ defined by

$$
(-1)^{m} A(v, u)=-\int_{D} v M u d x+\sum_{j=0}^{m-1} \oint_{\partial D} N_{j}(v) Q_{j}(u) d s
$$

is a symmetric positive semidefinite bilinear form.

We shall be concerned in this paper with the following two initial-boundary value problems defined on the region $D \times[0, T)$ :

Problem I:

$$
\begin{aligned}
\rho(\partial u / \partial t) & =(-1)^{m} M u \text { in } D \times[0, T), \\
Q_{j}(u) & =(-1)^{m} f_{j}\left(N_{j} u\right), \quad j=0,1, \ldots, m-1 \text { on } \partial D \times[0, T), \\
u(x, 0) & =u_{0}(x), \text { and }
\end{aligned}
$$

\section{Problem II:}

$$
\begin{aligned}
\rho\left(\partial^{2} u / \partial t^{2}\right) & =(-1)^{m} M u \quad \text { in } D \times[0, T), \\
Q_{j}(u) & =(-1)^{m} f_{j}\left(N_{j} u\right), \quad j=0, \cdots, m-1 \text { on } \partial D \times[0, T), \\
u(x, 0) & =u_{0}(x), \quad(\partial u / \partial t)(x, 0)=v_{0}(x) .
\end{aligned}
$$

Here $\rho$ and $M$, as well as $Q_{j}$ and $\dot{N}_{j}$, are assumed to be independent of the parameter $t$. The function $\rho$ is assumed to be positive in $D$, and the $f_{j}$ are prescribed functions which we require to be locally integrable. In addition they are to satisfy for some positive $a$, for a constant $p$ to be specified later, and for all $m-1$ times continuously differentiable functions $\phi$ the condition

$$
\sum_{j=0}^{m-1}\left\{N_{j}(\phi) f_{j}\left(N_{j}(\phi)\right)-2(p \alpha+1) \int_{0}^{N_{j}(\phi)} f_{j}(\sigma) d \sigma\right\} \geq 0 .
$$

It is easily checked that if for arbitrary $Z_{j}$ the point functions $f_{j}$ are of the form

$$
f_{j}\left(Z_{j}\right)=\left|Z_{j}\right|^{2 p a+1} h_{j}\left(Z_{j}\right),
$$


where each $b_{j}$ is a nondecreasing function of $Z_{j}$, then $(1.5)$ is satisfied automatically.

The purpose of the present note is to show that no solution with initial data in a certain class can exist for all time provided (1.5), with $p$ appropriately chosen, is satisfied. The method used to establish these results is a concavity method which has been employed in the recent literature (see, e.g., Levine [2], [3], Levine and Payne [4] and Knops, Levine and Payne [1]).

2. Problem I. In this section we establish the following theorem:

Theorem I. No solution of problem I, whose initial data satisfy the inequality

$$
\sum_{j=0}^{m-1} \oint_{\partial D} \int_{0}^{j N_{j}\left(u_{0}(s)\right)} f_{j}(\eta) d \eta d s>1 / 2 A\left(u_{0}, u_{0}\right)
$$

can exist for all time if (1.5) is satisfied for $p=1$.

To apply the concavity method we must find a twice continuously differentiable nonnegative functional $F(t)$ defined on solutions of problem I such that

$$
\begin{array}{r}
F(t)=0=, u=0, \\
\text { (2) } d^{2} F^{-a / d t^{2} \leq 0,} \quad a>0 .
\end{array}
$$

Here $\alpha$ will turn out to be the constant $\alpha$ in (1.5). Clearly, in order to establish $(2.2,(2))$ we need only show that $F F^{\prime \prime}-(\alpha+1)\left(F^{\prime}\right)^{2} \geq 0$.

To prove the theorem we first assume that $u$ exists on $D \times[0, \infty)$ and then show that this leads to a contradiction. We select an $F(t)$ of the following form for $0 \leq t \leq T_{0}<\infty$ :

$$
F(t)=\int_{0}^{t} \int_{D} \rho u^{2} d x d \eta+\left(T_{0}-t\right) \int_{D} \rho u_{0}^{2} d x+\beta(t+\tau)^{2}
$$

for as yet undetermined positive constants $T_{0}, \beta$ and $\tau$. Then

$$
\begin{aligned}
\frac{d F}{d t} & =\int_{D} \rho u^{2} d x-\int_{D_{0}} \rho u_{0}^{2} d x+2 \beta(t+\tau) \\
(2.4) & =2 \int_{0}^{t} \int_{D} \rho u \frac{\partial u}{\partial \eta} d x d \eta+2 \beta(t+\tau)=2 \int_{0}^{t} \int_{D}(-1)^{m} u M u d x d \eta+2 \beta(t+\tau) \\
& =2 \sum_{j=0}^{m-1} \oint_{\partial D} \int_{0}^{t} N_{j}(u) f_{j}\left(N_{j}(u)\right) d s d \eta-2 \int_{0}^{t} A(u, u) d \eta+2 \beta(t+\tau)
\end{aligned}
$$


and

$$
\frac{d^{2} F}{d t^{2}}=2 \sum_{j=0}^{m-1} \oint_{\partial D} N_{j}(u) f_{j}\left(N_{j}(u)\right) d s-2 A(u, u)+2 \beta .
$$

We now form

$$
\begin{aligned}
& F \frac{d^{2} F}{d t^{2}}-(\alpha+1)\left(\frac{d F}{d t}\right)^{2} \\
& \geq F\left\{-2 A(u, u)+2 \sum_{j=0}^{m-1} \oint_{\partial D} N_{j}(u) f\left(N_{j}(u)\right) d s+2 \beta\right. \\
& \left.(2.6) \quad-4(\alpha+1) \int_{0}^{t} \int_{D} \rho\left(\frac{\partial u}{\partial \eta}\right)^{2} d x d \eta\right\} .
\end{aligned}
$$

We have dropped the term

$$
\left\{4(\alpha+1)\left(T_{0}-t\right) \int_{D} \rho u_{0}^{2} d x\right\}\left\{\int_{0}^{t} \int_{D} \rho\left(\frac{\partial u}{\partial \eta}\right)^{2} d x d \eta+\beta\right\}
$$

after using Schwarz's inequality in the expression for $(d F / d t)^{2}$. Now

$$
2 \int_{0}^{t} \int_{D} \rho\left(\frac{\partial u}{\partial \eta}\right)^{2} d x d \eta=2(-1)^{m} \int_{0}^{t} \int_{D} M u \frac{\partial u}{\partial \eta} d x d \eta
$$

$$
\begin{aligned}
= & 2 \sum_{j=0}^{m-1} \oint_{\partial D} \int_{0}^{t} \frac{\partial}{\partial \eta}\left(N_{j}(u)\right) f_{j}\left(N_{j}(u)\right) d s d \eta-2 \int_{0}^{t} A\left(u, \frac{\partial u}{\partial \eta}\right) d \eta \\
= & 2 \sum_{j=0}^{m-1} \oint_{\partial D}\left\{\int_{0}^{N_{j}(u)} f_{j}(\sigma) d \sigma\right\} d s-A(u, u)+A\left(u_{0}, u_{0}\right) \\
& -2 \sum_{j=0}^{m-1} \oint_{\partial D}\left\{\int_{0}^{N_{j}\left(u_{0}\right)} f_{j}(\sigma) d \sigma\right\} d s .
\end{aligned}
$$

The insertion of $(2.7)$ into $(2.6)$ thus leads to

$$
\begin{aligned}
& {\left[F \frac{d^{2} F}{d t^{2}}-(\alpha+1)\left(\frac{d F}{d t}\right)^{2}\right] F^{-1}} \\
& (2.8) \geq 2 \alpha A(u, u)+2\left\{\sum_{j=0}^{m-1} \oint\left[N_{j}(u) f_{j}\left(N_{j}(u)\right)-2(\alpha+1) \int_{0}^{N_{j}(u)} f_{j}(\sigma) d \sigma\right] d s\right\} \\
& \quad-4(\alpha+1)\left[1 / 2 A\left(u_{0}, u_{0}\right)-\sum_{j=0}^{m-1} \oint_{\partial D}\left\{\int_{0}^{N_{j}\left(u_{0}\right)} f_{j}(\sigma) d \sigma\right\} d s\right]-2(2 \alpha+1) \beta .
\end{aligned}
$$


By hypothesis the first two terms on the right are nonnegative, and since the data term is assumed to satisfy (2.1), we may select

$$
\beta=\frac{2(\alpha+1)}{2 \alpha+1}\left\{\sum_{j=0}^{m-1} \oint_{\partial D}\left\{\int_{0}^{N_{j}\left(u_{0}\right)} f_{j}(\sigma) d \sigma\right\} d s-1 / 2 A\left(u_{0}, u_{0}\right)\right\} .
$$

With this choice we obtain the desired inequality

$$
F\left(d^{2} F / d t^{2}\right)-(\alpha+1)(d F / d t)^{2} \geq 0
$$

which will lead to the nonexistence result stated in the theorem. The consequences of (2.10) have been thoroughly investigated in [1] and [2] so we merely sketch the arguments. From the definition (2.3) we have that $F(t)>0$ in $[0, T]$ so that

$$
d^{2}\left(F^{-\alpha}\right) / d t^{2} \leq 0
$$

This inequality integrates to

$$
F^{-a}(t) \leq F^{-a}(0)\left[1-\alpha\left(F^{\prime}(0) / F(0)\right) t\right], \quad 0 \leq t \leq T_{0},
$$

which shows that $F^{-a}(t)$ must decay to zero in finite time $T \leq F(0) / \alpha F^{\prime}(0)$ if $F^{\prime}(0)>0$ and $T_{0} \geq F(0) / \alpha F^{\prime}(0)$. But with our choice of $F$ we have $F^{\prime}(0)$ $=2 \beta r>0$. A little algebra shows that $T_{0} \geq F(0) / \alpha F^{\prime}(0)$ provided $\tau$ is chosen to satisfy

$$
\tau>\frac{1}{2}(\alpha \beta)^{-1} \int_{D} \rho u_{0}^{2} d x
$$

and $T_{0}$ is then taken so large that

$$
T_{0} \geq \beta r^{2}\left[2 \alpha \beta r-\int_{D} \rho u_{0}^{2} d x\right]^{-1}
$$

The escape time $T$ cannot exceed the minimum value of the right-hand side of (2.14), considered as a function of $\tau$. In fact, $T \leq\left(\alpha^{2} \beta\right)^{-1} \int_{D} \rho u_{0}^{2} d x$.

3. Problem II. We now establish the following theorem:

Theorem II. No solution of problem II, whose initial data satisfy the inequality

(3.1) $E(0) \equiv \frac{1}{2}\left[\int_{D} \rho v_{0}^{2} d x+A\left(u_{0}, u_{0}\right)\right]-\sum_{j=0}^{m-1} \int_{\partial D}\left[\int_{0}^{N_{j}\left(u_{0}\right)} f_{j}(\sigma) d \sigma\right] d s<.0$, can exist for all time if (1.5) is satisfied for $p=2$. 
In this case we choose

$$
F(t)=\int_{D} \rho u^{2} d x+\beta(t+\tau)^{2}
$$

with $\beta$ and $\tau$ again positive but unprescribed. Then

$$
\frac{d F}{d t}=2 \int_{D} \rho u \frac{\partial u}{\partial t} d x+2 \beta(t+\tau)
$$

and

$$
\begin{aligned}
\frac{d^{2} F}{d t^{2}} & =2 \int_{D} \rho\left(\frac{\partial u}{\partial t}\right)^{2} d x+2(-1)^{m} \int_{D} u M u d x+2 \beta \\
& =2 \int_{D} \rho\left(\frac{\partial u}{\partial t}\right)^{2} d x+2 \sum_{j=0}^{m-1} \oint_{\partial D} N_{j}(u) f_{j}\left(N_{j}(u)\right) d s-2 A(u, u)+2 \beta .
\end{aligned}
$$

Now if we define

(3.5) $E(t)=\frac{1}{2}\left[\int_{D} \rho\left(\frac{\partial u}{\partial t}\right)^{2} d x+A(u, u)\right]-\sum_{j=0}^{m-1} \oint_{\partial D}\left[\int_{0}^{N_{j}(u)} f_{j}(\sigma) d \sigma\right] d s$ then

$$
\begin{aligned}
\frac{d E}{d t} & =\int_{D} \rho \frac{\partial u}{\partial t} \frac{\partial^{2} u}{\partial t^{2}} d x+A\left(u, \frac{\partial u}{\partial t}\right)-\sum_{j=0}^{m-1} \oint_{\partial D} N_{j}\left(\frac{\partial u}{\partial t}\right) f_{j}\left(N_{j}(u)\right) d s \\
& =\int_{D} \frac{\partial u}{\partial t}\left[\rho \frac{\partial^{2} u}{\partial t^{2}}-(-1)^{m} M u\right] d x=0 .
\end{aligned}
$$

Thus

$$
E(t)=E(0), 2
$$

and (3.4) may be rewritten, using (1.5) with $p=2$, as

$\frac{d^{2} F}{d t^{2}} \geq 2 \int_{D} \rho\left(\frac{\partial u}{\partial t}\right)^{2} d x+4(2 \alpha+1) \sum_{j=0}^{m-1} \oint_{\partial D}\left\{\int_{0}^{N_{j}(u)} f_{j}(\sigma) d \sigma\right\} d s-2 A(u, u)+2 \beta$, and using (3.5) and (3.7) as (3.9) $\frac{d^{2} F}{d t^{2}} \geq 4(\alpha+1) \int_{D} \rho\left(\frac{\partial u}{\partial t}\right)^{2} d x+4 a A(u, u)-4(2 \alpha+1) E(0)+2 \beta$.

${ }^{2}$ For weak solutions, one imposes instead the inequality $E(t) \leq E(0)$ as an additional hypothesis. 
It then follows that

$$
\begin{aligned}
F \frac{d^{2} F}{d t^{2}}-(\alpha+1)\left(\frac{d F}{d t}\right)^{2} & \\
\geq & 4(\alpha+1)\left[\left\{\int_{D} \rho u^{2} d x+\beta(t+\tau)^{2}\right\}\left\{\int_{D} \rho\left(\frac{\partial u}{\partial t}\right)^{2} d x+\beta\right\}\right. \\
& \left.\left(\int_{D} \rho u \frac{\partial u}{\partial t} d x+\beta(t+\tau)\right)^{2}\right] \\
+ & 4 a F A(u, u)-2(2 \alpha+1)[2 E(0)+\beta] F .
\end{aligned}
$$

The first term on the right is nonnegative by Schwarz's inequality. If we choose

$$
\beta=-2 E(0)
$$

we again are led to the inequality $d^{2}\left(F^{-a}\right) / d t^{2} \leq 0$, and hence to the breakdown of solution in finite time provided $F^{\prime}(0)>0$. But

$$
F(0)=2\left\{\int_{D} \rho u_{0} v_{0} d x-2 E(0) r\right\},
$$

and it is clear that because of condition (3.1) the constant $\tau$ may be chosen so large that $F^{\prime}(0)>0$ (provided $\int_{D} \rho u_{0} v_{0} d x$ is finite).

4. Concluding remarks. As pointed out in the introduction, similar inequalities hold for appropriately defined weak solutions of problems I and II. It is also clear that if we had assumed $A(u, u)$ to be positive definite and to satisfy

$$
A(u, u) \geq k^{2} \int_{D} \rho u^{2} d x
$$

we could have obtained somewhat different results for problem II.

For instance, suppose that in problem II, $E(0) \leq 0, \beta$ is chosen to be zero, and (4.1) is satisfied. Then (3.10) leads to

$$
F\left(d^{2} F / d t^{2}\right)-(\alpha+1)(d F / d t)^{2} \geq 4 k^{2} \alpha F^{2}
$$

or

$$
d^{2} F^{-\alpha} / d t^{2} \leq-4 k^{2} \alpha F^{-\alpha}
$$

We show that (4.3), and hence the solution of our problem, cannot exist for all time.

If we assume the contrary and make the substitution 


$$
y=\frac{d F^{-\alpha} / d t}{F^{-a}}=-a \frac{d F / d t}{F}
$$

then (4.3) becomes

$$
d y / d t+y^{2}+4 k^{2} \alpha \leq 0
$$

which integrates to give

$$
\tan ^{-1}[y(t) / 2 k \sqrt{\alpha}] \leq \tan ^{-1}[y(0) / 2 k \sqrt{\alpha}]-2 k \sqrt{\alpha} t .
$$

But clearly this inequality cannot hold for all time since the right-hand side tends to minus infinity while the left-hand side remains bounded as $t$ tends to infinity. In fact the solution must break down at some finite time $T$ which satisfies

$$
T \leq(2 k \sqrt{\alpha})^{-1}\left\{\pi / 2+\tan ^{-1}[y(0) / 2 k \sqrt{\alpha}]\right\} \text {. }
$$

It is easily seen as in [1]-[4] that various other combinations of initial data assumptions will lead to "blow-up" of solutions in finite time.

The restriction that none of the differential operators should depend on $t$ can be relaxed somewhat as was done in [2] and [3].

Finally, we remark that our results can be extended to certain types of higher order systems of equations where the coupling occurs in the boundary conditions. See [4].

\section{BIBLIOGRAPHY}

1. R. A. Knops, H. A. Levine and L. E. Payne, Nonexistence, instability and growth theorems for solutions to an abstract nonlinear equation with applications in elastodynamics Arch. Rational Mech. Anal. (to appear).

2. H. A. Levine, Some nonexistence and instability theorems for formally parabolic equations of the form $P u_{t}=-A(u)+\Phi(u)$, Arch. Rational Mech. Anal. 51 (1973), $371-386$.

3. - Instability and nonexistence of global solutions to nonlinear wave equations of the form $P u_{t t}=-A u+\mathscr{F}(u)$, Trans. Amer. Math. Soc. 192 (1974), 1-22.

4. H. A. Levine and L. E. Payne, Nonexistence theorems for the heat equation with nonlinear boundary conditions and for the porous medium equation backward in time, J. Differential Equations (to appear).

DEPARTMENT OF MATHEMATICS, UNIVERSITY OF RHODE ISLAND, KINGSTON, RHODE ISLAND 02881

DEPARTMENT OF MATHEMATICS, CORNELL UNIVERSITY, ITHACA, NEW YORK 14850 